\title{
Self-Consistent Theory of Field Electron Emission from Superconductors
}

\author{
E.A. Litvinov and I.V. Uimanov \\ Institute of Electrophysics, Ural Division of Russian Academy of Sciences, 34 Komsomolskaya Street, \\ Ekaterinburg 620049, Russia
}

\begin{abstract}
The tunneling Hamiltonian method generalized to describe the field emission process has been proposed and investigated as an approach for solving the field emission problem, taking into account nonequilibrium phenomena occurring in the cathode. By means of an analysis of the kinetics of the electron subsystem of a superconductor it has been shown that the nonequilibrium state with excess quasiparticles, appearing with field electron emission, changes the superconducting properties of the cathode. The dependence of the superconducting gap on the field emission current has been revealed. This has made it possible to formulate general concepts of a self-consistent theory of field electron emission from superconductors.
\end{abstract}

\section{INTRODUCTION}

First attempts to create the theory of field electron emission from superconductors were made soon after the discovery of the BCS theory [1-4]. However, no all-inclusive and consecutive theoretical description of the emissive properties of superconductors is now available, which would allow a detailed analysis of the totality of microscopic processes under field emission conditions. It should be noted that a substantial contribution to the understanding of the techniques applied for solving this problem was made by Gadzuk [1] and Gogadze and Kulik [2]. Their generalization of the tunneling Hamiltonian method for a description of the phenomenon of field emission from a solid into vacuum has made it possible to formulate the problem and has offered the technique for its solution based on the quantum field theory and using the mathematical apparatus of the time-dependent perturbation theory. However, the general disadvantage of the existing approaches to the creation of a self-consistent theory of field emission from superconductors is the assumption that the superconducting cathode is in an equilibrium state in the process of field emission, as generally assumed for metals. While for metals in the absence of strong heating processes this assumption is quite justified, for superconductors it requires an additional analysis of the conditions under which it would be applicable. The influence of field emission on superconductors leads to the generation of quasiparticles, so their number is greater than in thermal equilibrium. Taking into account that the energy gap and the stability of the state may be very sensitive to the quasiparticle energy distribution, the shape of the energy distribution of quasiparticles is the most important parameter of the theory. Therefore, a correct determination of the quasiparticle distribution function is the fundamental task of the theory, since the nonequilibrium state occurring in a superconducting cathode may substantially change the properties of the cathode itself and even carry it to the normal state.

\section{THE PRINCIPAL FIELD EMISSION CHARACTERISTICS IN A NONEQUILIBRIUM STATE}

Here we examine the expressions for the field electron emission (FEE) current density $j_{s}$ and the total energy distributions of field-emitted electrons (TED) $J_{E q}$ generalized for a nonequilibrium state of the superconductor. To derive these expressions, the tunneling Hamiltonian method [5] proposed and investigated for solving the FEE problem $[1,2]$ was used. Within the framework of this approach, one can obtain the generalized formal end result for the FEE current $[1,2]$ : 


$$
j_{\mathrm{s}}=4 e \operatorname{Im} \sum_{k, q}\left|T_{k, q}\right|^{2} \int d \omega G_{\mathrm{S}}^{<}(k, \omega) A_{\mathrm{v}}(q, \omega)
$$

where $\left|T_{k, q}\right|^{2}$ is the square of the tunneling matrix element for the field emission case [1], $G_{\mathrm{s}}^{<}(\boldsymbol{k}, \omega)$ is the single particle thermodynamic Green functions for the superconductor, $A_{\mathrm{v}}(q, \omega)$ is the spectral weight function for the vacuum. Thus, to obtain the emission current for the cathode in a nonequilibrium state, it is necessary to calculate the Green function $G_{\mathrm{s}}^{<}(k, \omega)$ of the superconductor for the nonequilibrium case. As shown by Keldysh [6], the apparatus of Green functions for nonequilibrium systems make possible a direct transfer to the kinetic theory approach for the nonequilibrium distribution function of the quasiparticles. In accordance with Ref. 7., we use the relationship between the Green function $G_{\mathrm{S}}^{<}(k, \omega)$ for the nonequilibrium state of the superconductor and the nonequilibrium distribution function for the quasiparticles $n_{k}$ :

$$
G_{\mathrm{s}}^{<}(\mathbf{k}, \omega)=2 \pi i\left[\mathrm{v}_{\mathrm{k}}^{2}\left(1-n_{\mathrm{k}}\right) \delta\left(\omega+E_{\mathbf{k}}\right)+\mathrm{u}_{\mathrm{k}}^{2} n_{\mathrm{k}} \delta\left(\omega-E_{\mathrm{k}}\right)\right], \mathrm{v}_{k}^{2}=\frac{1}{2}\left(1-\frac{\xi}{E}\right), \mathrm{v}_{k}^{2}+\mathrm{u}_{k}^{2}=1
$$

where $v_{k}^{2}$ and $u_{k}^{2}$ are the BCS coherence factors, $\xi=\varepsilon_{k}-\mu_{s}$ is the electron energy relative to the chemical potential of the pairs ( $\mu_{s}$ equals $\varepsilon_{F}$ only in an equilibrium state ), $E=\sqrt{\xi^{2}+\Delta^{2}}$ is the excitation spectrum of the superconductor, and $\Delta$ is the superconducting energy gap. Taking into account that the vacuum spectral weight function is sharp, that is $A_{\mathrm{V}}(q, \omega) \rightarrow \delta\left(E_{q}-\omega\right)$, eq. (1) becomes

$$
j_{s}=4 \pi e \sum_{k, q}\left|T_{k, q}\right|^{2}\left[\mathrm{v}_{k}^{2}\left(1-n_{k}\right) \delta\left(E_{q}+E_{k}\right)+\mathrm{u}_{k}^{2} n_{k} \delta\left(E_{q}-E_{k}\right)\right] \text {. }
$$

Using the expression for the square of the tunneling matrix element for the field emission case [1] and performing in eq. (4) the summation over all initial $\left(k, k_{\perp}>0\right)$ and final $\left(q, q_{\perp}>0\right)$ states, we obtain the final expressions for the FEE current:

$$
j_{s}=\frac{j_{0}}{d} \exp \left[\frac{\delta \mu_{\mathrm{s}}}{d}\right] \int_{-\mu_{s}}^{\infty} d \xi\left[\mathrm{v}_{k}^{2}(1-n(\xi))+\mathrm{u}_{k}^{2} n(\xi)\right] \exp \left[\frac{\xi}{d}\right]=\frac{j_{0}}{d} \exp \left[\frac{\delta \mu_{\mathrm{s}}}{d}\right] \int_{-\mu_{\mathrm{s}}}^{\infty} d \xi\left[\mathrm{v}_{k}^{2}+\frac{\xi}{E} n(\xi)\right] \exp \left[\frac{\xi}{d}\right]
$$

and for the total energy distribution (TED) for $\left|E_{q}\right|>\Delta$ :

$$
\begin{aligned}
J_{E_{q}}=\frac{\partial j_{s}}{\partial E_{q}}=\frac{j_{0}}{d} \exp \left[\frac{\delta \mu_{\mathrm{s}}}{d}\right] \frac{\left|E_{q}\right|}{\sqrt{E_{q}{ }^{2}-\Delta^{2}}} \frac{1}{2}\left\{f_{\xi>0}\left(\left|E_{q}\right|\right) \exp \left(\frac{\sqrt{E_{q}^{2}-\Delta^{2}}}{d}\right)+f_{\xi<0}\left(\left|E_{q}\right|\right) \exp \left(-\frac{\sqrt{E_{q}{ }^{2}-\Delta^{2}}}{d}\right)\right. \\
\left.+\frac{\sqrt{E_{q}{ }^{2}-\Delta^{2}}}{E_{q}}\left[f_{\xi>0}\left(\left|E_{q}\right|\right) \exp \left(\frac{\sqrt{E_{q}{ }^{2}-\Delta^{2}}}{d}\right)-f_{\xi<0}\left(\left|E_{q}\right|\right) \exp \left(-\frac{\sqrt{E_{q}{ }^{2}-\Delta^{2}}}{d}\right)\right]\right\}
\end{aligned}
$$

where $f_{\xi>0}^{\xi<0}\left(\left|E_{q}\right|\right)=n_{\xi>0}^{\xi<0}\left(\left|E_{q}\right|\right)$ for positive $E_{q}$ and $f_{\xi>0}^{\xi<0}\left(\left|E_{q}\right|\right)=1-n_{\xi>0}^{\xi<0}\left(\left|E_{q}\right|\right)$ for negative $E_{q}, n_{\xi>0}\left(\left|E_{q}\right|\right)$ and $n_{\xi<0}\left(\left|E_{q}\right|\right)$ are the energy distribution functions of electron-like $(\xi>0)$ and hole-like $(\xi<0)$ excitations, respectively. Here $\delta \mu_{\mathrm{s}}=\mu_{\mathrm{s}}-\varepsilon_{\mathrm{F}}$ is the deviation of the chemical potential of the Cooper pairs, $d=\hbar e F / 2(2 m \varphi)^{\frac{1}{2}} t\left(\left(e^{3} F\right)^{\frac{1}{2}} / \varphi\right)$, and $j_{0}=\left(2 \pi m e d^{2} / h^{3}\right) \mathrm{e}^{-c}$ is the field emission current at $T=0$.

It should be noted that for an equilibrium case $\left(n_{k}=n_{\mathrm{FD}}=\left\{1+\exp \left[E /\left(k_{\mathrm{B}} T\right)\right]\right\}^{-1}, f_{\xi>0}^{\xi<0}\left(\left|E_{q}\right|\right)=n_{\mathrm{FD}}\left(E_{q}\right)\right.$ for all $E_{q}$ ) it is not difficult to obtain from eqs.(4) and (5) the results of Refs.1, 2. On the other hand, the above expressions offer the opportunity to investigate the influence of the nonequilibrium processes occurring in a superconducting cathode on its emissive properties. Thus, in the general case, within the framework of the self-consistent approach, it is necessary to calculate the nonequilibrium distribution function of quasiparticles $n(\xi)$, the superconducting gap $\Delta$ and $\delta \mu_{s}$ in the nonequilibrium state. 


\section{TRANSPORT PHENOMENA IN THE SUPERCONDUCTING CATHODE}

The treatment of nonequilibrium processes in superconductors requires a system of equations which simultaneously describe both the dynamic and the kinetic properties. In the quasiclassical approximation, the full set of equations describing the transport phenomena in the electron system of a superconductor is formulated in [7]. In the steady state this system is reduced to the equation for the superconducting gap and to the kinetic equation for the quasiparticle distribution function. We shall deal with an approximate cathode geometry with the radius at the apex of the tip $r_{0} \sim 0.1 \div 1 \mu \mathrm{m}$ and with the angle $\theta$ at the apex of the tip about $5^{\circ}$, which is illustrated by Fig. 1. The model on which the following calculations are based is a pure superconductor where the electrons interact with phonons, and where the impurity scattering length $l_{i m p}=v_{\mathrm{F}} \tau_{\text {imp }}$ is large. We will assume that the phonons are in an equilibrium state at definite temperature. In terms of quasiparticles, the FEE

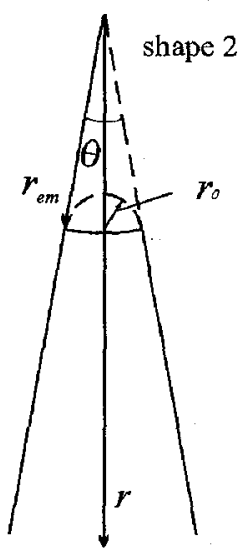

Fig. 1. The model cathode shapes. process can be treated as creation (or annihilation ) of quasiparticles at a short distance $\delta$ from the cathode surface $(\delta \approx 5 \AA$ ). Nonequilibrium excitations may then diffuse into the cathode from the emission surface over a characteristic diffusion length $L_{\varepsilon}$ which is $\left(v_{\mathrm{F}}{ }^{2} \tau_{\text {imp }} \tau_{\varepsilon} / 3\right)^{1 / 2}$, where $\tau_{\varepsilon}$ is the characteristic relaxation time due to electron-phonon collisions (for example, according to [8] for $\mathrm{Nb} \tau_{\varepsilon}=1.4910^{-9} \mathrm{c}$ ). Hence, as $L_{\varepsilon}$ $>r_{0}, \delta$, the model shapes of the cathode are workable and make it possible to use the one-dimensional approximation represented in Fig. 1 . In terms of the model chosen and in view of the assumptions made, using ordinary techniques, one may obtain from the kinetic equation for the quasiparticle distribution function the set of equations for $n_{0}$ and $n_{1}$ as follows [10]:

$$
\frac{|\xi|}{E} D \nabla^{2} n_{0}(\xi)=-\bar{I}_{p h}(n), \quad n_{1}=-l_{i m p} \operatorname{sign} \xi\left[\nabla n_{0}(\xi)\right]
$$

where $n_{0}$ and $n_{1} \mathrm{v} / \mathrm{v}$ are the first and the second term of the expansion of $\delta n$ in the Legendre polynomials, respectively, and $\delta n$ is the deviation from local equilibrium, and $\bar{I}_{p h}(n)$ is the angular average of the electron-phonon collision integral ( the full form of this integral can be found in [9,7] ). The equation for the energy gap in the nonequilibrium state is [7]

$$
1=\lambda \int_{0}^{\hbar \omega_{\mathrm{D}}} \frac{1-2 n_{k}}{\sqrt{\xi^{2}+\Delta^{2}}} d \xi \quad \text { or } \quad \Delta=\Delta_{0} \exp [-\bar{n}] \text {, where } \bar{n}=\int_{-\infty}^{\infty} \frac{n_{k}}{\sqrt{\xi^{2}+\Delta^{2}}} d \xi
$$

where $\lambda$ is the effective constant of the electron-electron coupling, and $\Delta_{0}$ is the energy gap at $T=0$. The latter is the well known equation for $\mathbf{p}_{\mathrm{s}}: \partial \mathbf{p}_{\mathrm{s}} / \partial t=-\nabla\left(\mu_{\mathrm{s}}+e \phi\right)$, where $\phi$ is the electrostatic potential. For the steady state, this equation becomes: $\quad \delta \mu_{\mathrm{s}}=-e \phi$

This formula correlates the change of the chemical potential associated with the pairs and with the electrostatic potential. To solve eq.(6), appropriate boundary conditions should be formulated. For this purpose, we obtain an expression for the quasiparticle flux $j_{q}^{e m}(\xi)$ through the emission surface in the energy range from $\xi$ to $\xi+d \xi$. We can demonstrate the derivation of $j_{q}^{\text {em }}(\xi)$ by means of Table 1 .

TABLE 1. Analysis of tunneling processes

\begin{tabular}{|l|c|c|c|}
\hline \multicolumn{1}{|c|}{ Processes } & Probability & $\begin{array}{c}\text { Electrons } \\
\text { removed }\end{array}$ & $\begin{array}{c}\text { Quasiparticles } \\
\text { removed }\end{array}$ \\
\hline emission of electrons from pair states & $\mathrm{v}_{k}^{2}\left(1-n_{k}\right)$ & 1 & -1 \\
\hline emission of electrons from excitation states & $\mathrm{u}_{k}^{2} n_{k}$ & 1 & 1 \\
\hline
\end{tabular}


Table 1 summarizes the tunneling processes with FEE based on eq. (3). According to this table, the expression for $j^{q} \mathrm{em}(\xi)$ can be written as

$$
j_{q}^{e m}(\xi)=\frac{j_{0}}{e d} \exp \left[\frac{\delta \mu_{s}}{d}\right]\left(\mathrm{v}_{\mathbf{k}}^{2}\left(1-n_{0}(\xi)\right)-\mathrm{u}_{\mathbf{k}}^{2} n_{0}(\xi)\right) \exp \left[\frac{\xi}{d}\right]=\frac{j_{0}}{e d} \exp \left[\frac{\delta \mu_{\mathrm{s}}}{d}\right]\left(\mathrm{v}_{\mathbf{k}}^{2}-n_{0}(\xi)\right) \exp \left[\frac{\xi}{d}\right]
$$

As seen from eq. (9), with FEE hole-like excitations are basically created over a wide range of energies $\sim d$ and, hence, the FEE process leads to a disbalance in the populations of the electron- and hole-like spectrum branches of excitations. According to eq.(6), the quasiparticles flux in the superconductor in the energy range from $\xi$ to $\xi+d \xi$ is $j_{q}(\xi)=-l_{i m p} N p_{\mathrm{F}}^{-1} \nabla n_{0}(\xi)$, where $p_{\mathrm{F}}=m v_{\mathrm{F}}$ and $N$ is the electron concentration. Then the boundary condition at the cathode surface can be written as follows:

$$
\left.j_{q}(\xi, r)\right|_{\mathrm{r}=\mathrm{r}_{\mathrm{cos}}}=j_{q}^{e m}(\xi) ; \quad L_{\varepsilon} \nabla n_{0}\left(\xi, r_{e m}\right)=v \tau_{\mathrm{\varepsilon}}\left(\mathrm{v}_{k}^{2}-n_{k}^{0}\right) \exp \left(\frac{\xi}{d}\right) \equiv \alpha\left(\mathrm{v}_{k}^{2}-n_{k}^{0}\right) \exp \left(\frac{\xi}{d}\right),
$$

where $v=j d\left(e d 2 N(0) L_{\mathrm{s}}\right)$ can be defined as the characteristic tunnel frequency in the FEE case, $2 N(0)$ is the density of states at the Fermi level and $\alpha=v \tau_{\varepsilon}$ can be defined as the relative intensity of the source of the nonequilibrium quasiparticles. Note that $\alpha$ is a function of $j_{0} / d$ for given parameters of the cathode material and its change will be associated with the variation of the applied electric field in our consideration.

\section{Analytical solutions in the temperature region close to $\mathbf{T}_{c}$}

First, using the neutrality condition, one obtains $\delta \mu_{s}=\int d \xi(\xi / E) n_{0}[12]$. For $T \rightarrow T_{c}$, it is possible to obtain an analytical solution of eqs.(6) in the time relaxation approximation. The integral of the electronphonon collisions can be linearized in this case $[10,11]$ and one can obtain an equation for $\delta \mu_{s}$ :

$L_{\varepsilon}^{2} \nabla^{2} \delta \mu_{s}-\left(\tau_{\varepsilon} / \tau_{q}\right) \delta \mu_{s}=0$, with boundary conditions: $L_{\varepsilon} \nabla \delta \mu_{s}\left(r=r_{e m}\right)=-\alpha d, \quad \delta \mu_{s}(r \rightarrow \infty)=0$,

where $\tau_{q}=\tau_{\varepsilon} 4 k_{\mathrm{B}} T /(\pi \Delta)$ is the charge relaxation time [11]. The solutions of eq. (11) for the model cathode shapes we obtain in the form:

cylindrical shape: $\delta \mu_{\mathrm{s}}=\alpha d \frac{l_{\mathrm{F}}}{L_{\varepsilon}} \exp \left(-\frac{r}{l_{\mathrm{F}}}\right) ; \quad$ conical shape: $\delta \mu_{\mathrm{s}}=\alpha d \frac{l_{\mathrm{F}}}{L_{\varepsilon}} \frac{r_{\mathrm{e}}^{2}}{l_{\mathrm{F}}+r_{e m}} \frac{1}{r} \exp \left(\frac{r_{e m}-r}{l_{\mathrm{F}}}\right)$,

where $l_{\mathrm{F}}$ is the penetration depth of the electric field into the cathode and $l_{\mathrm{F}} / L_{\varepsilon}=\left(4 k_{\mathrm{B}} T /(\pi \Delta)\right)^{1 / 2}$. Thus, the odd part of $n^{o}$ in $\xi, n_{0}^{o d d}$, associated with branch imbalance determines the shift of the condensate chemical potential $\mu_{s}$ and, hence, the electric field $\phi$ (see eq.(8)). Then the expression for $n_{0}{ }^{\text {odd }}$ in the linear approximation can be written as $[12,13]$

$$
n_{0}^{o d d}=-\delta \mu \frac{\xi}{E} \frac{\partial n_{F D}}{\partial E}=\frac{\delta \mu_{s}}{\gamma} \frac{\xi}{E} \frac{\partial n_{F D}}{\partial E}, \quad \text { where } \gamma=\left.\int d \xi\left(\frac{\xi}{E}\right) 2 \frac{\partial n_{F D}}{\partial E}\right|_{T \rightarrow T_{c}}=1-\frac{\pi \Delta(T)}{4 k_{\mathrm{B}} T}
$$

Hence, the results obtained by the linear analysis for $T \rightarrow T_{c}$ lead to the following conclusions. The main part of the nonequilibrium distribution function of the quasiparticles associated with branch imbalance has no effect on the gap magnitude, but determines the depth of penetration of the electric field into the cathode (similar to the penetration of the electric field when current flows through a metal-superconductor boundary $[10,11])$. As shown in [12], the even part of $n_{0}$ in $\xi, n_{0}{ }^{\text {even }}$, and the nonequilibrium gap magnitude $\Delta$ associated with branch imbalance can be written for the first order of $\Delta / T$ in the form:

$$
n_{0}^{e v e n}=\frac{\delta \mu_{s}^{2}}{4\left(k_{\mathrm{B}} T\right)^{2}} \operatorname{th}\left(\frac{E}{2 k_{\mathrm{B}} T}\right) \operatorname{ch}^{-2}\left(\frac{E}{2 k_{\mathrm{B}} T}\right) ; \quad \Delta=\sqrt{\Delta(T)^{2}-2 \delta \mu_{s}^{2}},
$$

where $\Delta(T)$ is the temperature-dependent BCS energy gap. Combining eqs. (12) and (14), one can obtain an equation for the energy gap $\Delta$ versus emission current.

\section{Numerical solution}

In this section we discuss the results we have obtained by solving numerically a system of coupled nonlinear equations. The quasiparticle kinetic equation (6) together with the gap equation (7) were solved 
for various values of $\alpha$ (associated with various values of the applied electric field, see eq.(10)) at several temperatures, taking into account the boundary conditions eq.(10). Here we present the results for $\mathrm{Nb}$ cathode. However, the procedure of seeking for a solution by itself suggests that the qualitative picture will be the same for other ( at least normal metallic) superconductors. The behavior of the nonequilibrium quasiparticle distribution function at the cathode surface is illustrated by Fig. 2 . First of all, note the branch imbalance between the electron-like and hole-like excitations that does occur in all nonequilibrium situation. All excess excitations are basically concentrated in the energy range $\sim T$. This decreases the superconducting gap (see Fig. 3.) and, hence, the critical parameters of the superconductor. The dependencies of the energy gap $\Delta$ and $\delta \mu_{s}$ versus distance $r$ from the cathode surface are shown in Fig. 4 . As illustrated in Fig.4, the penetration depth of the electric field $\left(e \phi=-\delta \mu_{s}\right)$ is large and increases with $T$ $\rightarrow T_{c}$. Increasing $\theta$ for the cone-shaped cathode enhances the efficiency of the diffusive mechanism of relaxation of the nonequilibrium quasiparticles. Note that an increase of the FEE current substantially increases the degree of nonequilibrium and may cause the cathode to lose its superconducting properties.

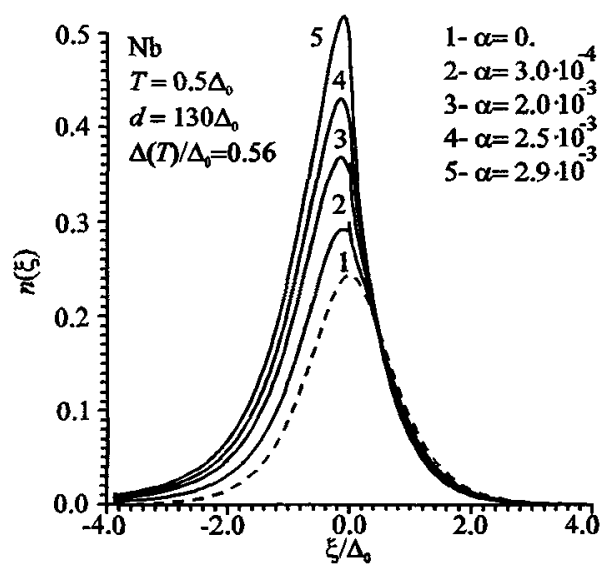

Fig. 2. Energy distribution function of quasiparticles at the cathode surface.

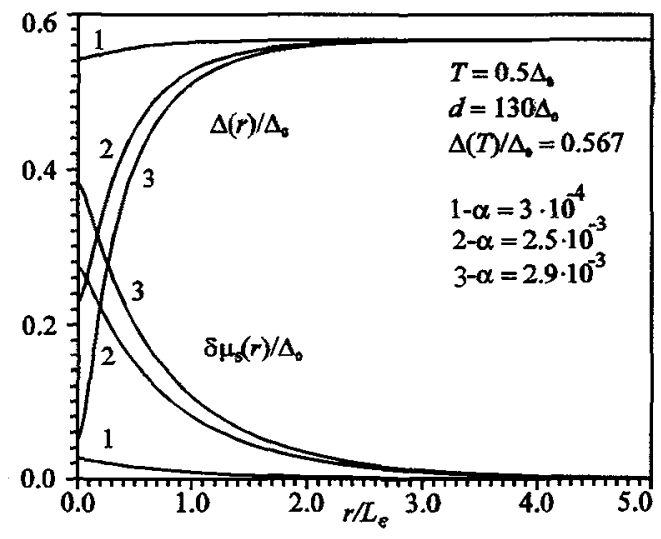

Fig. 4. Energy gap $\Delta$ and $\delta \mu_{\text {s }}$ as functions of distance $r$ from the cathode surface.

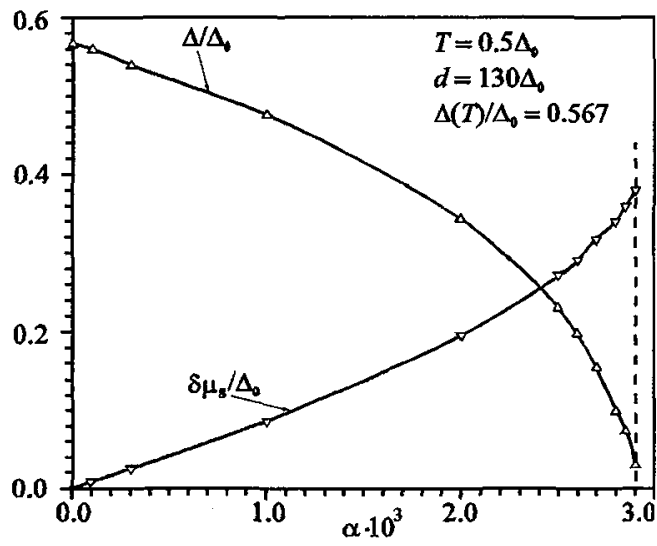

Fig. 3.Energy gap $\Delta$ and $\delta \mu_{s}$ at the cathode surface as functions of $\alpha$.

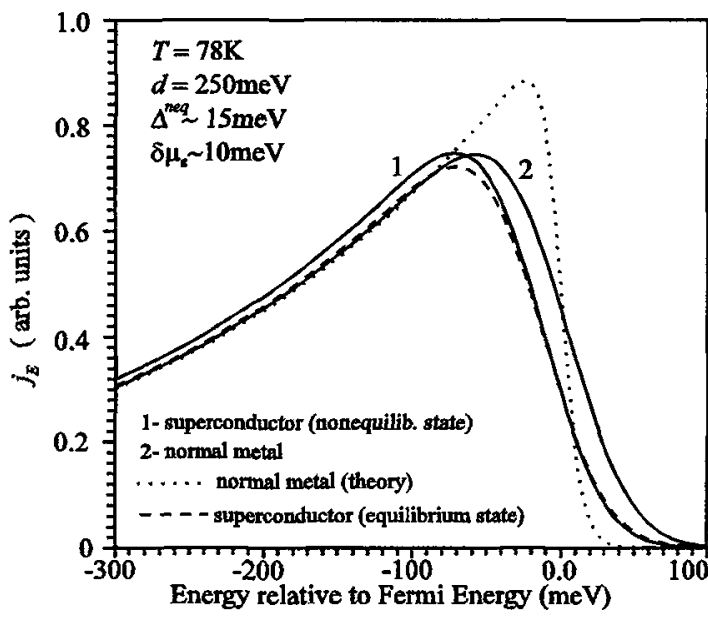

Fig. 5. TEDs with the limited resolution effect[15] ( $\Delta E_{1 / 2}=80 \mathrm{meV}$ ) for a normal metal and for a high- $T_{\mathrm{c}}$ superconductor. 


\section{THE EFFECT OF THE NONEQULIBRIUM CATHODE STATE ON THE TED}

Using eqs.(13) and (5), the effect of the branch imbalance on the TED can be described as

$$
\begin{aligned}
J_{E_{q}}^{\text {neq }}=\frac{j_{0}}{d} \exp \left[\frac{\delta \mu_{\mathrm{s}}}{d}\right] & \left\{\frac{\left|E_{q}\right|}{\sqrt{E_{q}{ }^{2}-\Delta^{2}}} \frac{1}{1+\exp \left(E_{q} / k_{\mathrm{B}} T\right)}\left[\cosh \left(\frac{\sqrt{E_{q}{ }^{2}-\Delta^{2}}}{d}\right)+\frac{\sqrt{E_{q}{ }^{2}-\Delta^{2}}}{E_{q}} \sinh \left(\frac{\sqrt{E_{q}{ }^{2}-\Delta^{2}}}{d}\right)\right]\right. \\
& \left.-\frac{\delta \mu_{\mathrm{s}} \operatorname{sigh}\left(E_{q}\right)}{4 k_{\mathrm{B}} T \cosh ^{2}\left(\frac{E_{q}}{2 k_{\mathrm{B}} T}\right)}\left[\sinh \left(\frac{\sqrt{E_{q}{ }^{2}-\Delta^{2}}}{d}\right)+\frac{\sqrt{E_{q}{ }^{2}-\Delta^{2}}}{E_{q}} \cosh \left(\frac{\sqrt{E_{q}{ }^{2}-\Delta^{2}}}{d}\right)\right]\right\}
\end{aligned}
$$

According to eq.(15), the branch imbalance effect leads to a shift of TED as a whole to the lower energies (about $\delta \mu_{\mathrm{s}} \approx \Delta$ ). Thus, an experimental determination of this shift for normal superconductors could be a problem as a result of the resolution limitations of present day experimental equipment. However, note that a similar energy shift was observed in [13] in studying the TED for the high- $T_{c} 2223 \mathrm{BiSrCaCuO}$ superconductor at $T<T_{c}$. The authors of this paper explained this TED shift by the existence of a gap $2 \Delta$ in the TED and by the relatively low resolution of the analyzer. However, in our opinion, the gap $2 \Delta \approx 50$ $\mathrm{meV}$ in the high energy tail of the TED cannot cause a uniform shift of the whole TED, taking into account that the full width of the TED is about $400 \div 600 \mathrm{meV}$. At the same time, many nonequilibrium phenomena observed in high- $T_{c}$ superconductors are conceptually similar [14]. Figure 5 presents TEDs for a normal metal and for a high- $T_{c}$ superconductor ( with approximated parameters: $\Delta \approx 15 \mathrm{meV}, d / \Delta_{0} \approx 5$ ) obtained taking into account the limited resolution of the energy analyzer [15]. It can be seen that the behavior of the TEDs agrees well with the experimental data [13]. Hence, one can suggest that the energy shift observed in [13] is associated with the branch imbalance between the electron-like and hole-like excitations in the nonequilibrium state appearing with field electron emission.

\section{CONCLUSION}

Thus, the above analysis of the kinetics of the electron subsystem of a superconductor allows one to investigate both the influence of the nonequilibrium state of the superconductor on the emission characteristics and the influence of the FEE process on the superconducting properties of the cathode and to draw the conclusion that excess quasiparticles created by FEE may cause the cathode to lose its superconducting properties. At the same time, the performed study opens up prospects for the investigation of fundamental properties of superconductors by field emission methods and offers a way for controlling the superconducting properties by varying the FEE current. This can be used in the development of various devices whose operation is based on the properties of nonequilibrium superconductivity.

\section{REFERENCES}

1. J. W. Gadzuk, Surf. Sci. 15466 (1969).

2. G. A. Gogadze, I. O. Kulik, Fiz. Met. Metaloved. 23 (1967) 606 (in Russian).

3. A. Leger, J. de Physique (Paris) 29 (1968) 648.

4. R. Klein and L. B. Leder, Phys. Rev. 124 (1961) 1050.

5. M. H. Cohen, L. M. Falicov and J. C. Phillips, Phys. Rev. Letters 8 (1962) 316.

6. Keldysh, L. V., 1964, Zh. Eksp. Teor. Fis. 47, 1515.

7. A. G. Aronov and V. L. Gurevich, Zh. Eksp. Teor. Fiz., 65 (1973) 1111.

8. J. J. Chang find D. J. Scalapino, 1977, J. Low Temp. Phys. 29, 477.

9. G. M. Eliashberg, 1970, Zh. Eksp. Teor. Fiz. Pis'ma, 11, 186.

10. Artemenko, S. N., A. F. Volkov and A. V. Zaitsev, 1978, J. Low Temp. Phys. $30,487$.

11. C. J. Pethick, H. Smith, J. Phys. C, 13 (1980) 6313.

12. B. I. Ivlev, Zh. Eksp. Teor. Fiz. 75, 5 (1978) 1771.

13. N. Ernst, W. A. Schmidt, Ch. Kleint, A. J. Melmed and G. L. Larkins, Physica C 213 (1993) 495-499.

14. Dmitriev, V. M., Zolochevskii, I. B. and Khristenko, E. V., Fiz. Nizk. Temp., v. 19, 3 (1993) 249.

15. R. D. Young and C. E. Kuyatt, Rev. Sci. Instr. 39 (1968) 1477. 